\title{
5-Alpha-Reductase Inhibitor Treatment for Frontal Fibrosing Alopecia:A Myth or Truth?
}

\author{
Hsuan-Hsiang Chen* \\ Department of Dermatology, National Taiwan University Hospital and National Taiwan University College of Medicine, Taipei, Taiwan
}

\author{
Received: September 03, 2021; Accepted: September 20, 2021; Published: September 27, 2021 \\ *Corresponding author: Hsuan-Hsiang Chen, MD,Department of Dermatology, National Taiwan University Hospital, 7, Chung-Shan South \\ Road,Taipei, Taiwan, Tel. No: +886-2-2356-2141; Fax. No: +886-2-2393-4177, E-mail: beauty101@gmail.com
}

Frontal Fibrosing Alopecia (FFA) is a condition described in 1994 affect mainly postmenopausal women. It is the most common form of cicatricial alopecia considered as a follicular variant of lichen planopilaris [1]. It is believed that the main pathogenesis in this condition is related to dihydrotestosterone, a derivative converted from the testosterone through 5-AlphaReductase (5AR). Because inflammatory, autoimmune, and other organisms are thought to be associated with FFA, there is no one specific cause that can be acted on by 5AR inhibitors [2].Currently, the therapeutic approaches are limited and the patient outcomes are poor [1]. To date, most of the reports are case reports and observational studies [3]. No randomized controlled studies are evaluating the pharmacodynamic profiles of $5 \mathrm{AR}$ inhibitors for FFA. The long-term safety of 5AR inhibitors in women should also be considered.

Murad et al. endorsed the use of finasteride or dutasteride for the treatment of FFA by a meta-analysis of observational studies and case reports between 2005 and 2017 [3]. They graded these reports with the American College of Physicians outcome study grading system [4]. In their article, 14 papers based on using $5 \mathrm{AR}$ inhibitors to manage FFA were evaluated to grade the safety and efficacy of this treatment. Among them, 121 patients were treated with finasteride and 149 subjects with dutasteride. The dose of finasteride was between 2.5 to $5 \mathrm{mg}$ daily, and that of dutasteride was $0.5 \mathrm{mg}$ once weekly, respectively. Although FFA is more common in women, the article did not specify whether outcomes with 5 AR inhibitors were similar in men. Their research revealed that the use of 5AR inhibitors showed the disease stabilized or less progressed. Very low numbers showed hair regrowth, especially with dutasteride. However, whether dutasteride is superior to finasteride is unknown. The research by Donovan et al. as well as Gamret et al. also showed clinical improvement with 5 AR inhibitors [5,6]. However, none of the literature selected was from a grade 1 randomized double-blinded controlled study, which made these analyses at risk of multiple confounding factors, including the presence of co-existing androgenetic alopecia, concurrent treatment use with topical minoxidil and topical or intralesional corticosteroids, limited follow-up duration period, and the possibility of spontaneous disease remission or stabilization.

Recently, Imhof et al. conducted a review of the literature to evaluate the treatment options available for FFA [7]. Their result was similar to Murad's study. Porriño-Bustamante et al. performed an updated and complete review about FFA from 1994 to 2021. Their results also confirmed Murad's study results [8]. Other reviews by Esteban-Lucia and Molina included Vañò's original multicenter study, just like the study by Murad et al. $[9,10]$. However, none of the studies included in these reviews analyzing the efficacy of 5AR inhibitors were randomized controlled studies.

Regarding the side effect of 5AR inhibitors, the safety profiles of the treatment couldn't be determined as most studies did not report on this aspect. Seale et al. confirmed the occurrence of decreased libido in postmenopausal women treated with finasteride [11]. In considering the risk of breast cancer, only 4 patients were included in the study by Murad et al. [3]. Their analyses did not report any possible outliers, such as genetic predisposition to breast cancer, concurrent use of other highrisk medications, previous history of breast cancer, etc. The same shortcomings are also noted in the aspects of reduced libido as well as increased psychiatric illnesses.

In conclusion, 5AR inhibitors are most effective in treating FFA when used in combination with other treatments in postmenopausal women. It is inconclusive in premenopausal women due to a lack of research on teratogenicity. When used as the sole treatment, 5AR inhibitors worked most effectively in stabilizing the disease and were less effective in improving the disease.Currently, the empirical evidence is of low quality and subjective. 5AR inhibitors are generally used as an adjunct rather than a primary treatment option. It is suggested that FFA be treated with 5AR inhibitors before systemic immunosuppressive agents if conventional treatment is not successful. Further doubleblinded randomized controlled studies to find the best treatment for FFA should be considered as it causes cicatricial alopecia with a great impact on the psychological status of the patient.

\section{References}

1. Cranwell WC, Sinclair R. Familial frontal fibrosing alopecia treated with dutasteride, minoxidil and artificial hair transplantation. Australas J Dermatol. 2017;58(3):e94-e96.

2. Tavakolpour S, Mahmoudi H, Abedini R, Hesari K, Kiani A, 
Daneshpazhooh M. Frontal fibrosing alopecia: An update on the hypothesis of pathogenesis and treatment. Int J Womens Dermatol. 2019;5(2):116-23.

3. Murad A, Bergfeld W. 5-alpha-reductase inhibitor treatment for frontal fibrosing alopecia: an evidence-based treatment update. J Eur Acad Dermatol Venereol. 2018;32(8):1385-90.

4. Qaseem A. The development of clinical practice guidelines and guidance statements of the American College of Physicians: Summary of methods. Ann Intern Med. 2010;153(3):194-199.

5. Donovan J. Finasteride-mediated hair regrowth and reversal of atrophy in a patient with frontal fibrosing alopecia. JAAD Case Rep. 2015;1(6):353-355

6. Gamret A, Potluri V, Krishnamurthy K, Fertig, R. Frontal fibrosing alopecia: efficacy of treatment modalities. Int J Womens Health. 2019;11:273-285.

7. Imhof R, Tolkachjov S. Optimal management of frontal fibrosing alopecia: A practical guide. Clin Cosmet Investig Dermatol
2020;13:897-910

8. Bustamante ML, Pugnaire MA, Santiago S. Frontal fibrosing alopecia: A review. J Clin Med 2021;10(9):1805.

9. Lucía L, Ruiz AM, Requena L. Actualización en alopecia frontal fibrosante. Actas Dermosifiliogr. 2017;108(4):293-304.

10. Galván S, Ruiz AM, Falcón C, Santiago S, Barata AR, Saucedo G, et al Frontal fibrosing alopecia: A multicenter review of 355 patients. J Am Acad Dermatol. 2014;70(4):670-678.

11. Seale DS, Eglini BA, Micheal A. Side effects related to 5-alphareducatase inhibitor treatment of hair loss in female: A review. J Drugs Dermatol. 2016;15(4):414-419. 